\title{
Bilateral Avascular Necrosis of the Femoral Head in a Patient with Asymptomatic Adrenal Incidentaloma
}

\author{
Joon-Soo Ha, MD, Hong-Man Cho, MD*, Hyun-Ju Lee, MD*, Sun-Do Kim, MD* \\ Department of Orthopaedics, King's College Hospital, London, UK \\ Department of Orthopedic Surgery, Gwangju Veterans Hospital, Gwangju, Korea*
}

Except when caused by direct and definite mechanisms (e.g., injury of the vessels to the femoral head), the pathophysiology of avascular necrosis of the femoral head has not yet been fully elucidate. While non-traumatic avascular necrosis of the femoral head is known to be caused by alcohol, steroids and various diseases, it may also occur without such events in a patient's history. Herein, a case of bilateral avascular necrosis of the femoral head caused by asymptomatic adrenal cortex incidentaloma which was initially misdiagnosed as idiopathic is reported along with a literature review.

Key Words: Adrenal gland neoplasms, Cushing's syndrome, Femur head necrosis

Avascular necrosis (AVN) of the femoral head is the result of decreased intramedullary blood flow from a number of potential causes. A specific risk-factor for non-traumatic AVN of the femoral head is a history of drug use ${ }^{1}$. Steroids, which are reported to contribute to $10 \%$ to $30 \%$ of all cases of AVN of the femoral head', and Cushing's syndrome, a state of endogenous cortisol excess, are also reportedly associated with this condition ${ }^{3,4)}$. Adrenal incidentaloma (AI) is a benign asymptomatic

Submitted: February 22, 2019 1st revision: April 19, 2019

Final acceptance: April 22, 2019

Address reprint request to

Hong-Man Cho, MD

(https://orcid.org/0000-0001-5160-2640)

Department of Orthopedic Surgery, Gwangju Veterans Hospital,

99 Cheomdanwolbong-ro, Gwangsan-gu, Gwangju 62284, Korea

TEL: +82-62-602-6162 FAX: +82-62-602-6936

E-mail: chm1228ahanmail.net

This is an Open Access article distributed under the terms of the Creative Commons Attribution Non-Commercial License (http://creativecommons. org/licenses/by-nc/4.0) which permits unrestricted non-commercial use, distribution, and reproduction in any medium, provided the original work is properly cited. adenoma that is often found incidentally during abdominal imaging, but may progress to symptomatic Cushing's syndrome ${ }^{5)}$. Technological advances in imaging have increased the diagnosis rate of AI. The authors experienced a case where a patient with asymptomatic AI developed Cushing's syndrome an initial surgery. The patient underwent bilateral total hip arthroplasty (THA) after being diagnosed with bilateral idiopathic AVN of the femoral head. We report the case with a review of the literature.

\section{CASE REPORT}

A 36-year old female patient visited our hospital with a chief complaint of pain and restricted range of motion (ROM) in the left hip joint persisting for two months. Physical examination of the patient revealed severe gait disturbance secondary to hip pain aggravated by hip joint flexion or rotation. The patient had no lifestyle habits or specific comorbidities thought to cause femoral head AVN. An anteroposterior view of the left hip revealed sclerosis and collapse of the femoral head and dysplasia of the hip (Fig. 1A). The magnetic resonance imaging (MRI) scan 


\section{Hip \& Pelvis}

Joon-Soo Ha et al. Avascular Necrosis of the Femoral Head due to Adrenal Incidentaloma

revealed an increased amount of joint fluid and bone marrow edema in the left hip, and femoral head necrosis was detected on the contralateral side; however, the patient did not complain of any pain (Fig. 1B). The patient underwent THA after being diagnosed with idiopathic osteonecrosis of the femoral head, was discharged in good condition three weeks after the surgery without specific complications, and followed up via regular outpatient visits. The patient complained of pain and limited ROM in the contralateral hip joint and gait disturbance one year after the initial surgery. Her symptoms continued for two months and increased over the following three weeks. Repeat MRI revealed similar findings to those noted previously in the left hip (Fig. 1C). A second THA was performed (one year after the first THA of the left hip); the patient was discharged in good condition after the surgery without specific complications and followed up via regular outpatient visits (Fig. 1D). At the time of the second surgery, the patient was $7 \mathrm{~kg}$ heavier than she was at the time of the first procedure and developed a moderate moon face, signs that were initially overlooked as weight gain. Subsequently, the patient complained of intensifying feelings of helplessness and central obesity and muscle mass reduction in both the upper and lower limbs aggravated over a three-month period of outpatient follow-up after the second surgery. However, these signs
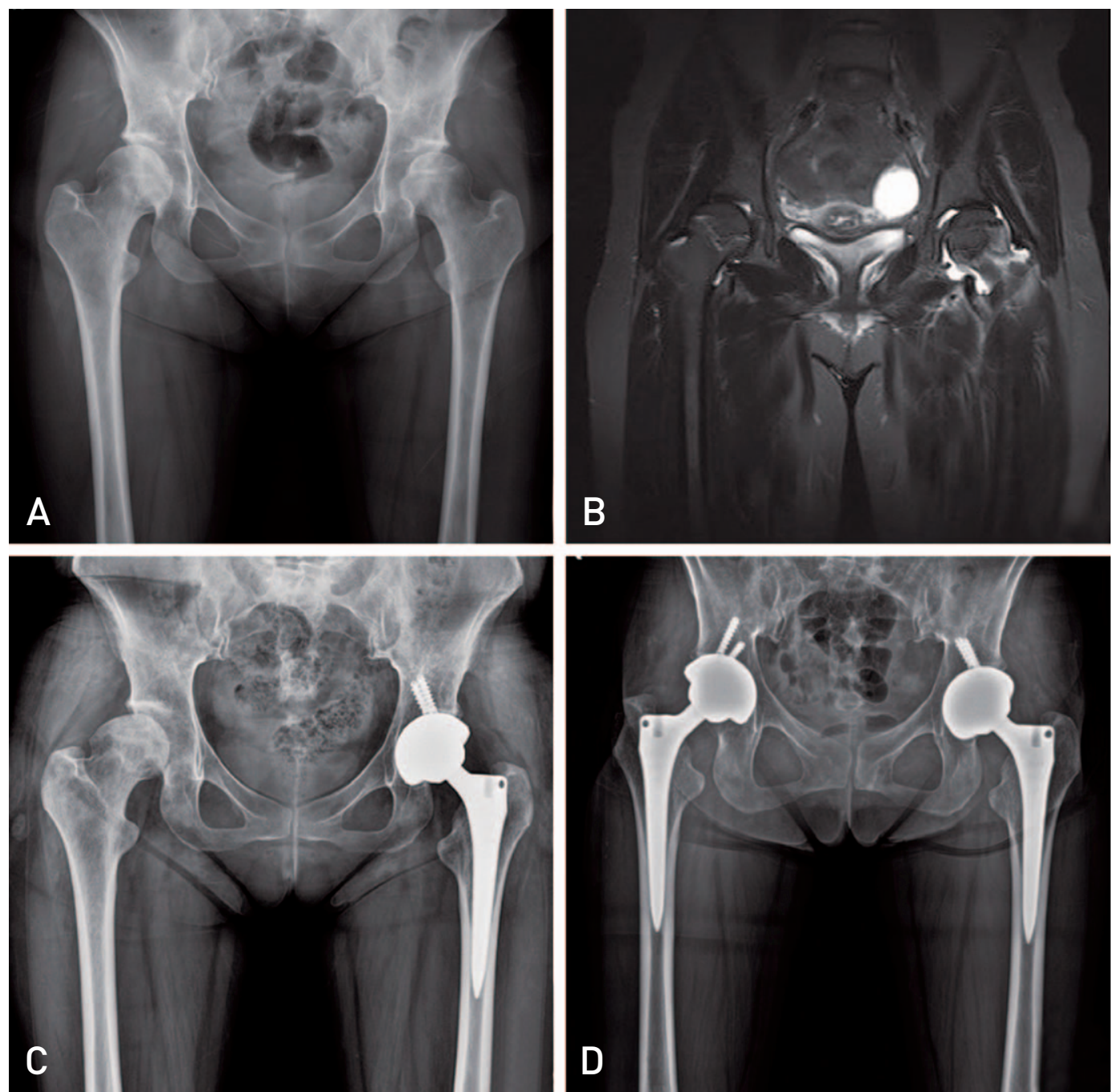

Fig. 1. Femoral head necrosis in a 36-year-old female patient. (A) Anteroposterior view of the right femoral head reveals areas of hyperlucency and surrounding sclerosis, as well as subtle changes in the shape of the articular surface. (B) Magnetic resonance imaging of the hip joint. Bilateral femoral head osteonecrosis, noted to be more extensive in the left hip. (C) Radiograph 12 months after initial examination reveals severe joint destruction of the right femoral head (D) and following total hip arthroplasty. 


\section{Hip \& Pelvis}

Hip Pelvis 31(2): 120-123, 2019

were not significant enough to warrant further investigation. One year after the second surgery, the patient was referred to the Department of Cardiology in our hospital due to a progressive worsening of central obesity and muscle mass reduction of the limbs and hot flushes. She was diagnosed with hypertension due to high blood pressure and electrolyte inbalance and prescribed medication. She was suspected of having secondary hypertension, and abdominal computed tomography (CT) and echocardiography were conducted. Although echocardiography yielded no specific findings, abdominal CT detected a $2.5 \mathrm{~cm}$ benign adrenal adenoma (Fig. 2A). The patient was immediately referred to the Department of Endocrinology to be screened for Cushing's syndrome. During screening, a 24-hour urine free cortisol revealed an elevation to $394.3 \mu \mathrm{g} /$ day (normal, $>50 \mu \mathrm{g} /$ day) and a plasma cortisol level of $29.0 \mu \mathrm{g} / \mathrm{dL}$ (normal, $<5.0$ $\mu \mathrm{g} / \mathrm{dL}$ ) was noted based on the results of a 48-hour lowdose dexamethasone suppression test. A plasma adrenocorticotropic hormone (ACTH) of $21.5 \mathrm{pg} / \mathrm{mL}$ (normal range, 6-76 pg/mL) and cortisol levels between 17.3 and $19.9 \mu \mathrm{g} /$ day in a high-dose dexamethasone test confirmed Cushing's syndrome. A suspected cause was ectopic ACTH-producing tumors due to an adrenocortical adenoma. An in-depth history was then undertaken. The patient disregarded advice to undergo regular monitoring of a small abdominal mass found in a regular check-up done several years before her first visit to our hospital. At the time, she had menstrual irregularity, mild weight gain, depression and intermittent facial skin problems-clinical signs associated with Cushing's syndrome. We determined that asymptomatic AI developed into symptomatic Cushing's syndrome, and the patient underwent laparoscopic partial adrenalectomy after referral to our urology department (Fig. 2B). Histopathological examination confirmed cortical adenoma. Six months after the procedure, Cushing's syndrome-related symptoms completely disappeared, including feelings of helplessness and central obesity. The patient is currently under outpatient observation without any specific symptoms.

The patient's informed consent and institutional review board's clearance were obtained for the purpose of publication of a case report. This article was approved by Gwangju Veterans Hospital at July 2, 2018 (project number, GVHIRB 18-0023).

\section{DISCUSSION}

The long-term or high-dose use of steroids may cause AVN of the femoral head; steroid use has been identified to contribute to $10 \%$ to $30 \%$ of cases of femoral head $\mathrm{AVN}^{6}$. A large-scale study on steroid-related AVN of the femoral head suggested that $3(4 \%)$ of 77 patients were related to endogenous Cushing's syndrome ${ }^{7}$. The etiology of AVN of the femoral head caused by hypercortisolism in patients with endogenous Cushing's syndrome has not been fully clarified, but a number of hypotheses concerning pathogenesis exist. First, a theory suggests that fat embolization from the liver, bone marrow fat cells, or destabilization and coalescence of plasma lipoproteins might be responsible for the pathogenesis. Another possible cause is hypertension and arteriosclerosis secondary to corticosteroids inducing insulin resistance which may
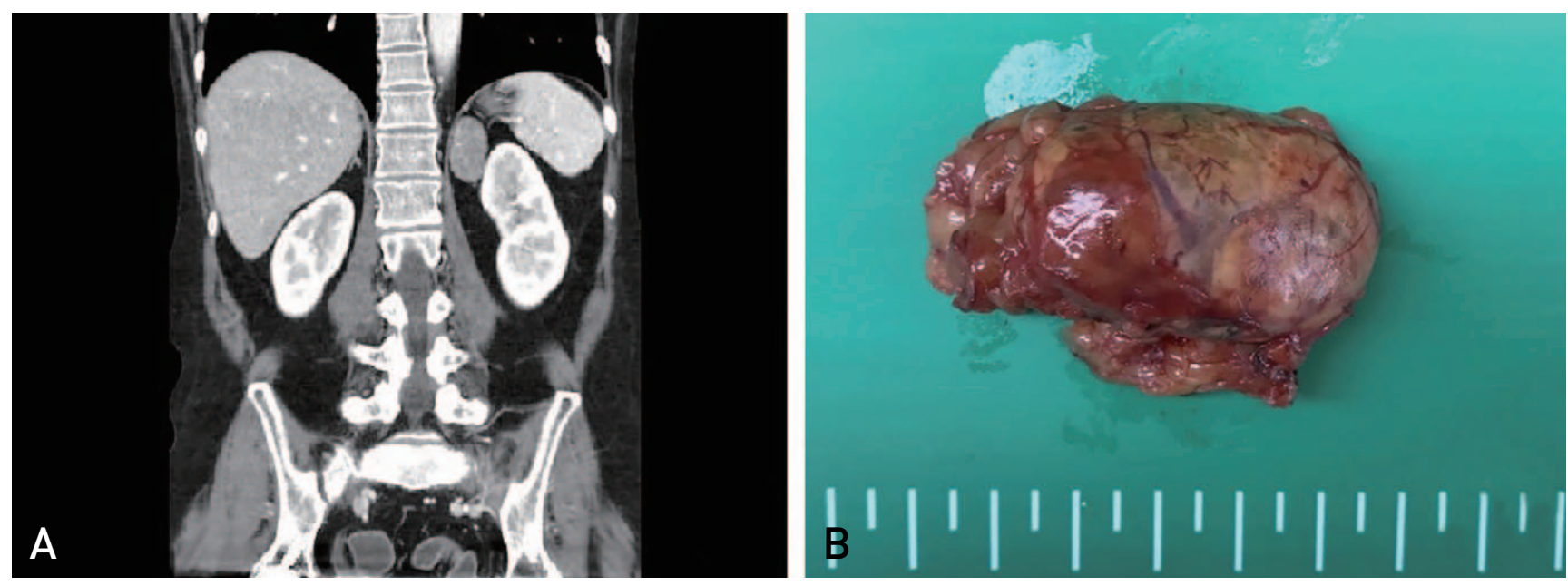

Fig. 2. (A) A homogeneous mass with a smooth border, prompt washout and an attenuation value of less than 10 Hounsfield units on unenhanced computed tomography. (B) Gross photograph of the cut surface of the adrenal gland. The tumor, which is $2.0 \mathrm{~cm}$ in diameter, is composed of interspersed yellow and brown areas. 
lead to AVN. For this reason, AVN is also called a "coronary disease of the hip" ${ }^{\text {') }}$. Another possible factor is microfractures caused by osteoporosis induced by exposure to excessive corticosteroids.

AIs, often found incidentally, are mostly asymptomatic and inactive masses; recent advances in imaging technology have increased the frequency of detection ${ }^{5}$. The majority of AIs are non-cortisol-secreting and non-functioning masses, but have recently drawn much attention because these masses are associated with a risk of developing functioning masses with cortisol hypersecretion and may eventually progress to the state of affecting the hypothalamicpituitary-adrenal axis (in roughly $12-16 \%$ of patients with $\mathrm{AI})^{9}$.

Like the case presented here, various problems (e.g., AVN) may occur when asymptomatic AI increases endogenous cortisol production following the development of functioning masses. However, since the risk of excessive cortisol production can be underestimated in patients with AI, a careful endocrine evaluation for cortisol levels is recommended ${ }^{10)}$.

The clinical symptoms of Cushing's syndrome vary substantially depending on the degree and duration of hypercortisolism. Muscle atrophy in the proximal musculature, increased amount of fat in the abdomen, chest and face, muscle loss in the upper and lower limbs, and wide purple striae suggest marked hypercortisolism, and these signs are suggestive of Cushing's syndrome. However, early diagnosis may be difficult because patients with Cushing's syndrome may not show typical symptoms and its signs may be considered "ordinary" physical changes. In relation to the occurrence of a bony lesion (e.g., femoral head osteonecrosis due to Cushing's syndrome), Cerletty et al. ${ }^{4}$ noted that patients with idiopathic osteonecrosis of the femoral head require careful monitoring because some of these cases are associated with endogenous hypercorticolism and may be overlooked. In particular, since non-traumatic osteonecrosis of the femur mainly occurs in younger, more active and working persons, more attention is warranted for joint preservation and patient's quality of life.

Approximately $25 \%$ of cases with femoral head osteonecrosis are classified as idiopathic. The early detection of asymptomatic Cushing's syndrome may help prevent associated complications, in particular, AVN of the femoral head that seriously affects patient's quality of life. When idiopathic osteonecrosis of the femoral head is suspected in young patients, like the patient in this case report, appropriate management is important including further investigation into the specific cause(s) of osteonecrosis.

\section{CONFLICT OF INTEREST}

The authors declare that there is no potential conflict of interest relevant to this article.

\section{REFERENCES}

1. Boettcher WG, Bonfiglio M, Smith K. Non-traumatic necrosis of the femoral head: Part II. Experience in treatment. J Bone Joint Surg. 1970;52:322-29.

2. Wang GJ, Dughman SS, Reger SI, Stamp WG. The effect of core decompression on femoral head blood flow in steroid-induced avascular necrosis of the femoral head. $J$ Bone Joint Surg Am. 1985;67:121-4.

3. Koch CA, Tsigos C, Patronas NJ, Papanicolaou DA. Cushing's disease presenting with avascular necrosis of the hip: an orthopedic emergency. J Clin Endocrinol Metab. 1999;84:3010-2.

4. Cerletty JM, Ziebert AP, Mueller KH. Avascular necrosis of the femoral head as the presenting manifestation of Cushing's disease. Clin Orthop Relat Res. 1973;(97):69-73.

5. Rossi R, Tauchmanova L, Luciano A, et al. Subclinical Cushing's syndrome in patients with adrenal incidentaloma: clinical and biochemical features. J Clin Endocrinol Metab. 2000;85:1440-8.

6. Wang GJ, Cui Q, Balian G. The pathogenesis and prevention of steroid-induced osteonecrosis. Clin Orthop Relat Res. 2000;(370):295-310.

7. Fisher DE, Bickel WH. Corticosteroid-induced avascular necrosis. A clinical study of seventy-seven patients. J Bone Joint Surg Am. 1971;53:859-73.

8. Hungerford DS, Zizic TM. Pathogenesis of ischemic necrosis of the femoral head. The hip. St. Louis: CV Mosby; 1983. 249-62.

9. Ambrosi B, Peverelli S, Passini E, et al. Abnormalities of endocrine function in patients with clinically "silent" adrenal masses. Eur J Endocrinol. 1995;132:422-8.

10. Terzolo M, Osella G, Alì A, et al. Subclinical Cushing's syndrome in adrenal incidentaloma. Clin Endocrinol (Oxf). 1998;48:89-97. 